\title{
It is all about the quality of the data
}

\author{
Robert J. Cerfolio, MD, MBA
}

\begin{abstract}
From the Department of Cardiothoracic Surgery, New York University Langone Health, New York, NY. Disclosures: Dr Cerfolio serves as a consultant for Intuitive, C-SATS, Bovie, Ethicon, Covidien/Medtronic, Community Health Services, Davol/Bard, Myriad Genetics, KCI, and Verb Medical.

Received for publication Oct 31, 2017; accepted for publication Nov 9, 2017; available ahead of print Dec 6, 2017. Address for reprints: Robert J. Cerfolio, MD, MBA, Director of Lung Cancer Service Line, Langone Medical Center, New York University (NYU), Chief of Clinical Division of Cardiothoracic Surgery, Department of CT Surgery, 550 First Ave, New York, NY, 10016 (E-mail: robert.cerfolio@nyumc.org).

J Thorac Cardiovasc Surg 2018;155:1344

$0022-5223 / \$ 36.00$

Copyright (C) 2017 by The American Association for Thoracic Surgery

https://doi.org/10.1016/j.jtcvs.2017.11.009
\end{abstract}

Jung and colleagues ${ }^{1}$ have presented an interesting article on an important clinical problem, postoperative delirium. The keys to any study's validity are the methods used, the validity of the data collected, and the definitions used. I have several concerns about some of these in this study. An outstanding statically analysis, as so nicely applied and executed by Jung and colleagues, ${ }^{1}$ cannot, unfortunately, validate inaccurate or poorly defined data points.

As Jung and colleagues ${ }^{1}$ point out in their article, "delirium was defined by using the Confusion Assessment Method (CAM), which was defined as positive during the ICU [intensive care unit] stay, or when haloperidol was administered within 4 days after surgery (CAM data were not available in ward)." In addition, Jung and colleagues ${ }^{1}$ tell us that "CAM has four features: (1) an acute onset of mental status changes or a fluctuating course; (2) inattention; (3) disorganized thinking; and (4) an altered level of consciousness. The patient is diagnosed as delirious (ie, CAM positive) if he or she has both features 1 and 2 and either feature 3 or 4." I believe that these criteria by themselves carry a high degree of observer variable and disagreement.

Jung and colleagues ${ }^{1}$ go on to state that " $[\mathrm{t}] \mathrm{rained}$ ICU nurses assessed delirium every hour until ICU discharge. Delirium was assessed for the first 4 days postoperatively in an effort to exclude delirium originating from surgical complications such as pneumonia, acute lung injury, sepsis, or conduit leak, which often occur after postoperative 3 or 4 days." This, too, would seem to inject severe and subjective observer variability. Yet only 1 nurse made the diagnosis and served as the criterion standard for the diagnosis.

The ability to judge a patient objectively with the criteria described by the Jung and colleagues ${ }^{1}$ remains dubious to

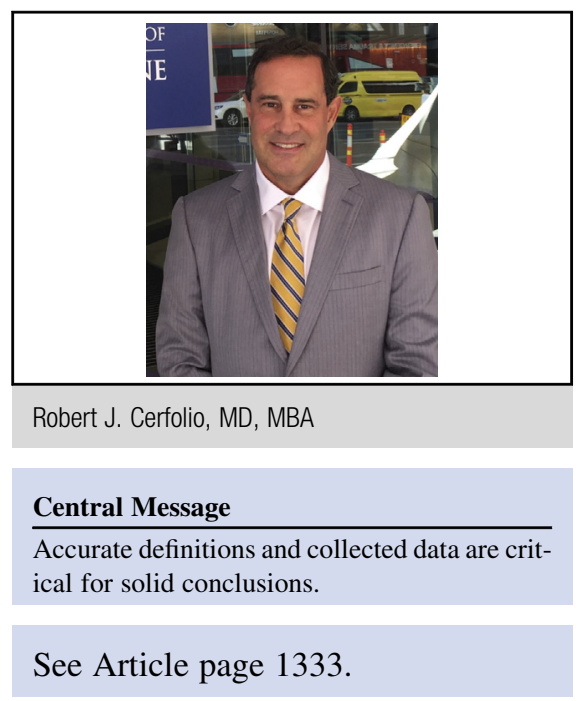

me. Even more damaging is the fact that these data were not collected on the floor. These facts, joined with the retrospective nature of the study and the exceedingly unusual and perhaps antiquated practices of keeping patients intubated overnight on the day of surgery and given continuous remifentanil, both of which necessitate the use the routine use of the ICU, make the data even more difficult to interpret, and perhaps less applicable to many of our clinical practices in North America.

I in no way mean to disparage Jung and colleagues. ${ }^{1}$ In fact, I congratulate them for the fantastic surgical results of this series: 1041 esophagectomies over 6 years with good presented outcomes. This is a challenging group of complex patients. In addition, Jung and colleagues ${ }^{1}$ point out some of these, as well as other limitations of this study, with refreshing self-awareness. Most of all, I congratulate Jung and colleagues ${ }^{1}$ on getting their study accepted and published in the Journal.

\section{Reference}

1. Jung DM, Ahn HJ, Yang M, Kim JA, Kim DK, Lee SM, et al. Hydroxyethyl starch is associated with early postoperative delirium in patients undergoing esophagectomy. J Thorac Cardiovasc Surg. 2018;155:1333-43. 